\title{
SISTEMATIZAÇÃO DA ASSISTÊNCIA DE ENFERMAGEM E 0 CÂNCER DE MAMA ENTRE MULHERES
}

\author{
Ana Carolina Almeida Pereiral \\ Déborah Viana Oliveira II \\ Smalyanna Sgren da Costa Andrade ${ }^{\text {III }}$
}

\section{RESUMO}

O câncer de mama tem grande incidência mundial e está cada vez mais presente em nosso cotidiano. Ele consiste no crescimento desordenado das células, que expande para o tecido e órgãos. Este estudo objetivou avaliar o efeito de intervenção educativa sobre o câncer de mama por meio da Sistematização da Assistência de Enfermagem (SAE). Trata-se de uma descrição da experiência de uma prática de saúde bem sucedida em Unidade de Saúde da Família, alocada em uma comunidade do município de Cabedelo, Paraíba. Para a descrição dos resultados foram seguidas as etapas da Classificação Internacional da Prática de Enfermagem $(\mathrm{CIPE})^{\circledR}$, através dos sete eixos. Considerando a importância da promoção da saúde, da disseminação dos meios de prevenção e detecção precoce do câncer de mama, esta proposta fortaleceu a autonomia profissional em atividades educativas através do uso da SAE.

\section{PALAVRAS-CHAVE}

Mulher. Climatério. Aspectos biopsicossociais. Fitoterapia.

\section{INTRODUÇÃO}

O câncer de mama tem grande incidência mundial e está cada vez mais presente em nosso cotidiano1. De acordo com dados do Instituto Nacional do Câncer (INCA), esse tipo de câncer perfaz cerca de $28 \%$ da ocorrência dos novos casos de câncer a cada ano, sendo o mais comum entre as mulheres no Brasil. Apesar de infrequente na população masculina, o câncer de mama também poderá acometer $1 \%$ dos homens ${ }^{2}$.

Sob outra ótica, o déficit de conhecimento relacionado ao câncer dificulta a busca pela assistência à saúde, mesmo com a manifestação de sinais e sintomas importantes como nódulo, eritema da mama, retração, alterações no mamilo e edema. A pouca importância dada às alterações, seja pela própria mulher ou seus pares, como família e amigos pode dificultar a detecção precoce, piorando a gravidade do problema3.

De acordo com a Organização Pan-Americana de Saúde e Organização Mundial de Saúde (OPAS/OMS), no ano de 2012 diagnosticaram-se cerca de 408 mil

\footnotetext{
I Acadêmica do Curso de Graduação de Enfermagem da Faculdade de Enfermagem Nova Esperança - FACENE. End.: Rua José Ayres de Lucena, 67, Ernesto Geisel. João Pessoa, Paraíba, Brasil. Cel.: (83) 98858-8903. E-mail: carolinaalmeidajp@gmail.com.

Il Acadêmica do Curso de Graduação de Enfermagem da Faculdade de Enfermagem Nova Esperança - FACENE, João Pessoa, Paraíba, Brasil.

III Enfermeira. Doutoranda do Programa de Pós-Graduação em Enfermagem da Universidade Federal da Paraíba e Docente do Curso de Graduação de Enfermagem da Faculdade de Enfermagem Nova Esperança - FACENE, João Pessoa, Paraíba, Brasil.
} 
mulheres, com a doença, e 92 mil mulheres morreram em consequência dela nas Américas. Até o ano de 2030, os novos casos podem aumentar em 46\%4. Em âmbito nacional, o Brasil possui altas taxas de incidência e mortalidade. No ano de 2013, o INCA, identificou que o número de mortes pelo câncer equivaleu a 14.388 , sendo 181 homens e 14.206 mulheres. Para o ano de 2018, houve a estimativa de 59.700 casos de ocorrência do câncer de mama com risco estimado de 56,33 casos a cada 100 mil mulheres 5 .

Na década de 90, como forma de estimular a população para a detecção precoce do câncer, foi criado o movimento popular e internacional intitulado Outubro Rosa, buscando promover a conscientização da população. Atualmente, é ofertado nesse período um maior acesso aos serviços de diagnóstico, cujo exame clínico das mamas e a mamografia se tornaram um dos meios mais eficazes de detecção, contribuindo para a redução da mortalidade ${ }^{6}$.

No que tange a prevenção, hábitos de vida saudáveis podem postergar o aparecimento do câncer de mama entre mulheres sem predisposição genética. A adoção de uma alimentação saudável aliada à realização de exercícios físicos promove a redução do risco de obesidade e sedentarismo. Além disso, a diminuição da ingestão de bebidas alcoólicas também pode ser um elemento preventivo relevante. Por isso, para prevenção da doença, a mulher deve adotar práticas de saúde satisfatórias7.

Nesse contexto de prevenção e detecção precoce, a Enfermagem se insere no âmbito da saúde como aliada poderosa à redução dos índices de acometidas. Denominada como a arte do cuidar, a Assistência de Enfermagem fornece um cuidado pleno, encorajador e comprometido em ajudar as mulheres, sejam acometidas por câncer ou para fins preventivos ${ }^{8}$.

No caso das acometidas, elas pre- cisam ter um tratamento individualizado e com um olhar holístico, devido às fragilidades dessa enfermidade. A influência da Enfermagem é de grande relevância, pois, atua de forma direta no cuidado, construindo vínculos, proporcionando conforto e desenvolvendo capacidade de superação dessas mulheres 9 .

Como forma de melhorar o processo de cuidado, conta-se com um método privativo do enfermeiro que éa Sistematização da Assistência de Enfermagem (SAE). Ela é utilizada em busca de um melhor plano e processo de trabalho, objetivando melhoria da assistência e a obtenção de resultados satisfatórios ${ }^{10}$.

A SAE é um instrumento constituído por cinco etapas. São elas: levantamento de dados, diagnósticos de enfermagem, planejamento, implementação e avaliação. Ela direciona e viabiliza o trabalho da equipe de enfermagem, auxilia os pacientes de acordo com as necessidades biopsicossociais e espirituais, através de suas habilidades, julgamento e raciocínio, melhorando a qualidade dos serviços prestados 8 .

Diante da percepção do aumento da incidência de novos casos, todo ano, e tendo em vista a importância da promoção da saúde, da disseminação dos meios de prevenção e detecção, faz-se necessário o uso da Sistematização da Assistência de Enfermagem (SAE) como forma de contribuir positivamente nesse processo de educação e conscientização da população. Justificam-se essas ações porque, a educação em saúde, sobre esse tema, deve ser algo contínuo, objetivando a efetividade do processo de cuidado.

Assim, esta proposta foi norteada pelo seguinte questionamento: A Sistematização da Assistência de Enfermagem (SAE) pode contribuir coma prevenção e detecção precoce do câncer de mama entre mulheres residentes em comunidade de ummunicípio paraibano? Por isso, estaproposta objetivou avaliar o efeito de intervenção educativa sobre o câncer demama pormeio daSAE. 


\section{METODOLOGIA}

Trata-se de uma descrição da experiência de uma prática de saúde bem sucedida em unidade de saúde da família, alocada em uma comunidade do município de Cabedelo, Paraíba. Para a descrição dos resultados foram seguidas as etapas da Classificação Internacional da Prática de Enfermagem $(\mathrm{CIPE})^{\circledR}$.

A CIPE ${ }^{\circledR}$ propõe sete eixos que podem ser implementados de acordo com a necessidade e conveniência do profissional ${ }^{11}$, quais sejam:

a) Foco: Área de atenção relevante para enfermagem.

b) ulgamento: Opinião clínica, determinação relacionada ao foco da prática profissional de enfermagem.

c) Meios: Forma ou método de con-

\section{RESULTADOS}

\section{Reconhecimento do ambiente}

No primeiro dia, a estrutura da unidade básica da comunidade em questão foi analisada para verificar a ambiência ideal, para a realização da intervenção em saúde. Havia escassez de recursos materiais, por isso qualquer estratégia deveria ser pensada para reduzir o máximo de despesas orçamentárias, considerando que o custeio seria resultado de financiamento próprio.

\section{da intervenção \\ Planejamento, organização e execução \\ O público-alvo da proposta foi consti-} tuído por dez mulheres e um homem. A intervenção foi planejada por seis acadêmicas da Faculdade de Enfermagem Nova Esperança, João Pessoa, Paraíba. A idealização da estratégia foi composta por elaboração de material impresso (folders) e uso de modelos anatômicos das mamas para identificação das manifestações clínicas, disponibilizados pela instituição de cretizar uma intervenção.

d) Ações: Processo intencional aplicado a um cliente ou desempenhado por um cliente.

e) Tempo: O ponto, período, instante, intervalo ou duração de uma ocorrência.

f) Localização: Orientação anatômica ou espacial de um diagnóstico ou intervenções.

g) Cliente: Sujeito a quem o diagnóstico se refere e que é o beneficiário de uma intervenção de enfermagem.

O período de execução da atividade ocorreu em junho de 2017. Os resultados foram expostos em forma de quadro e figura construída através do programa Cmap Tools ${ }^{\circledR}$, versão 6.01.01 e discutidos à luz da literatura.

ensino superior. Além disso, pensou-se em uma dinâmica de apresentação dos participantes por meio de uma roda de conversa.

As facilitadoras explicaram o motivo da presença delas no ambiente, apresentaram-se e em seguida sugeriram a exposição formal de cada participante. $A$ posteriori iniciaram uma discussão sobre as características do câncer de mama, manifestações clínicas e tratamento. Ao passo que a atividade técnica era realizada, havia o estímulo constante à participação de todos, com esclarecimento de dúvidas, desmistificação de mitos e tabus, bem como fortalecimento dos saberes individualizados.

Após a atividade técnica, houve a abertura de espaço para que houvesse o compartilhamento de experiências relacionadas ao câncer de mama, entre as mulheres da comunidade e família. Na ocasião, uma das participantes relatou a 
doença como algo vivenciado por ela, culminando em cirurgia e uma recidiva. O terceiro momento consistiu na avaliação sobre a efetividade da intervenção, cujas perguntas eram direcionadas aos participantes de maneira personalizada, havendo respostas condizentes com aquilo que foi compartilhado durante toda a ação. Por fim, houve agradecimentos e todos foram convidados para realização do lanche coletivo.

Descrição da Intervenção e a Classificação Internacional da Prática de Enfermagem (CIPE) ${ }^{\circledR}$

A Sistematização da Assistência de Enfermagem que norteou a ação, pode ser visualizada no quadro 1. Os eixos da CIPE ${ }^{\circledR}$ e os elementos da intervenção podem ser visualizados no quadro 2.

Quadro 1 - Descrição da intervenção conforme as etapas da Sistematização da Assistência de Enfermagem.

\begin{tabular}{l|l|}
\hline \multicolumn{1}{|c|}{ ETAPA } & \multicolumn{1}{c}{ ELEMENTOS } \\
\hline Levantamento dos dados & $\begin{array}{l}\text { 1. Avaliação da capacidade de aprendizado. } \\
\text { 2. Investigação sobre o conhecimento existente. }\end{array}$ \\
\hline Diagnóstico de Enfermagem & 1. Conhecimento baixo \\
\hline Planejamento & $\begin{array}{l}\text { 1. Roda de conversa: uso de folders, mamas anatômicas e } \\
\text { lanche coletivo. }\end{array}$ \\
\hline $\begin{array}{l}\text { 1. Estabelecimento de um ambiente de confiança e respeito } \\
\text { para estimulação do aprendizado. } \\
\text { 2. Compartilhamento de saberes técnicos sobre o processo } \\
\text { patológico com linguagem acessível. } \\
\text { 3. Orientação quanto aos meios de prevenção e deteç̧ão } \\
\text { precoce. } \\
\text { 4. Escuta qualificada das vivências. } \\
\text { 5. Estímulo ao autocuidado. }\end{array}$ \\
$\begin{array}{ll}\text { 1. Indagação sobre saberes técnicos compartilhados. } \\
\text { 2. Observação prática acerca da realização de açães de } \\
\text { autocuidado. } \\
\text { 3. Modificação do Diagnóstico de Enfermagem inicial. }\end{array}$ \\
\hline Avaliação
\end{tabular}

Quadro 2 - Eixos da Classificação Internacional da Prática da Enfermagem (CIPE®) conforme a intervenção em saúde planejada.

\begin{tabular}{|c|c|}
\hline EIXOS & ELEMENTOS DA INTERVENÇÃO \\
\hline Foco & Conhecimento sobre prevenção e detecção precoce \\
\hline Julgamento & Baixo \\
\hline Meios & Folders e prótese mamária \\
\hline Ações & Roda de conversa com compartilhamento de saberes e experiências \\
\hline Tempo & Sala de espera para consultas (duração: 1 hora) \\
\hline Localização & Anatômica: Mamas/ Geográfica: Unidade de Saúde \\
\hline Cliente & Mulheres e interessados da comunidade \\
\hline
\end{tabular}




\section{Desfecho da intervenção em saúde}

A estratégia trouxe resultados esperados à comunidade. A avaliação foi realizada por meio da observação situacional dos participantes, sendo evidenciado por rela- tos verbais ou feedbacks positivos quanto à ação. Adiante, é possível observar a comparação do Diagnóstico de Enfermagem Coletivo antes e após a intervenção com a respectiva definição contida na CIPE ${ }^{\circledR}$ (Figura 1).

Figura 1 - Definição do Foco e Julgamentos conforme a Classificação Internacional da Prática da Enfermagem (CIPE®) antes e após a intervenção planejada.

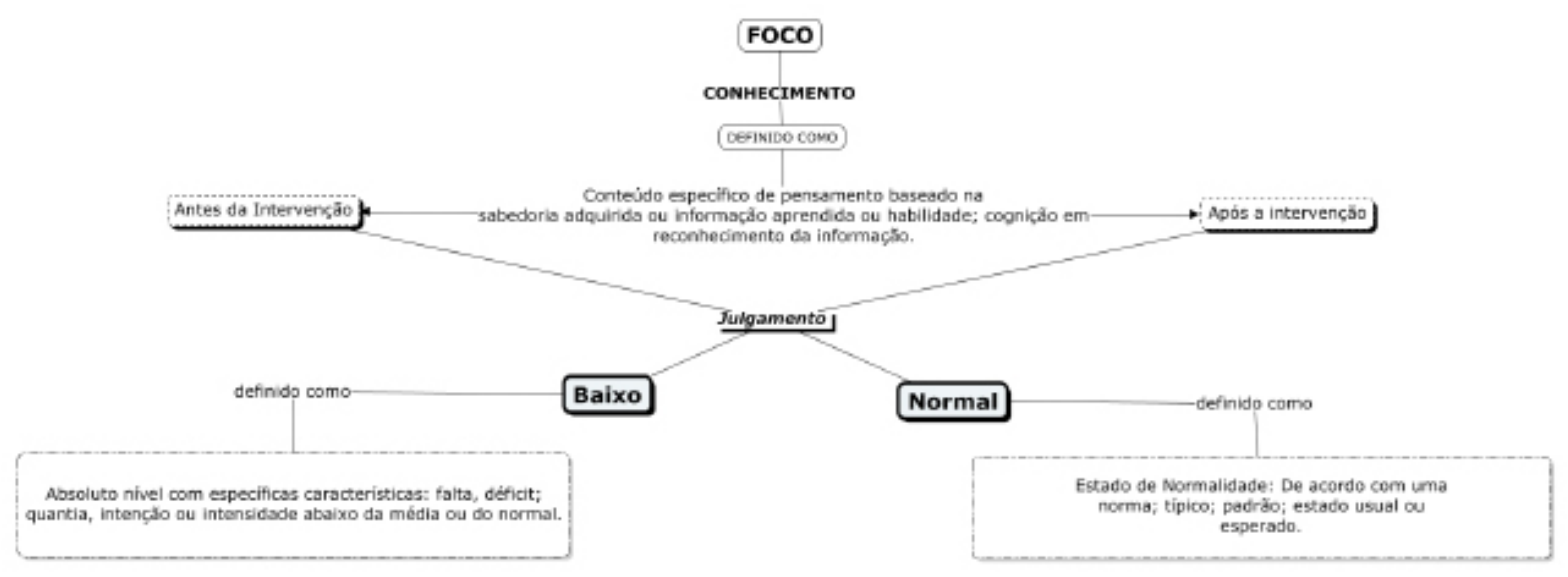

\section{DISCUSSÃO}

Ocâncer demama temalta prevalência na população de mulheres brasileiras. $O$ diagnóstico precoce e o rastreamento das manifestações clínicas são meios que favorecem o seu prognóstico favorável e aumentam as chances de cura da doença. A prevenção consiste na mudança de hábitos de vida como alimentação satisfatória associada ao abandono do tabagismo, etilismo e sedentarismo. Os meios de detecção precoce envolvem o autoexame, exame clínico das mamas e os exames de imagem ${ }^{12}$.

Conforme o autor supracitado, muitos tumores mamários são detectados pela mulher durante o autoexame das mamas, embora ele não haja consenso quanto à sua recomendação enquanto método para diagnóstico clínico. Apesar disso, percebe-se a relevância do autoexame das mamas para redução da mortalidade por este tipo de câncer.

Por isso, é importante a atuação do enfermeiro no incentivo às práticas de autocuidado, pois ele é um agente educador nato para compartilhamento de informações relacionadas ao câncer de mama. Além disso, o fato de haver maior percentual de mulheres nesta categoria trabalhista contribui para o envolvimento satisfatório, devido ao desenvolvimento da empatia pelo processo de adoecimento pelo câncer e posterior mastectomia ${ }^{13}$.

Por isso, estratégias educativas desenvolvidas pela enfermagem se constituem como práticas humanizadas para a promoção da saúde, prevenção de agravos e enfrentamento da doença. O impacto causado pela descoberta do câncer de mama e posterior mastectomia envolve aspectos não somente mutiladores do corpo, mas elementos psicossociais e uma rede interativa de apoio profissional, familiar e outros aparatos que merecem atenção 9 .

Logo, unir a educação em saúde, que faz parte do processo de cuidar da enfermagem à uma ferramenta que torna 
a categoria mais autônoma, como a Sistematização da Assistência de Enfermagem (SAE) é uma maneira de organizar, planejar e executar o cuidado com vistas à efetividade da assistência. No contexto da atenção básica, essa simbiose pode aumentar o diagnóstico precoce e reduzir os índices de mortalidade por câncer de mama na comunidade participante.

Nesse contexto, a Sistematização da Assistência de Enfermagem (SAE) está cada vez mais presente no cotidiano dos serviços e tornou-se uma exigência das instituições de saúde para melhoria do cuidado prestado ao indivíduo. Estudo indica a importância da enfermagem em todo o processo de educação em saúde, sendo primordial para obtenção do sucesso. A enfermagem encontra-se em todas as etapas pela qual o paciente, diagnosticado com câncer de mama passa, desde o diagnóstico até a reabilitação ${ }^{14}$.

Além disso, em busca de promover à saúde e proporcionar o autocuidado, a educação em saúde está tornando-se uma peça fundamental do processo de fortalecimento individual e coletivo em direção à promoção da saúde e prevenção de agravos. O processo de motivação quanto à participação da população por meio de rodas de conversas, debates e encontros, faz com que qualquer intervenção positiva se transforme no objeto primordial de empoderamento da população. Isso diminui os fatores que comprometam a saúde dos indivíduos ${ }^{15}$.

Pesquisa de revisão integrativa demonstrou que a educação em saúde voltada para o estímulo do autoexame das mamas não deve ser o único método de detecção precoce do câncer de mama, embora os autores afirmem que esta prática é necessária em comunidades vulneráveis devido ao aumento do autocuidado e do autoconhecimento da população feminina7.
No município de Piripiri-PI, através do Programa de Educação pelo Trabalho para a Saúde (PET-SAÚDE), estudantes de enfermagem e fisioterapia de uma instituição de ensino superior desenvolveram uma atividade educativa com mulheres usuárias da Unidade Básica de Saúde de dois bairros do município. A metodologia utilizada foi exposição dialogada por meio de rodas de conversas sobre a prevenção do câncer de mama. As mulheres eram questionadas quanto à prevenção e tratamento. Após ensinamentos sobre a temática com realização do autoexame das mamas (AEM), os facilitadores solicitaram que as participantes demonstrassem o que foi aprendido. Esse movimento de ensinar e aprender gerou retorno positivo, ao passo que as mulheres se sentiram protagonistas do seu próprio cuidado ${ }^{15}$.

Conforme o mesmo estudo, aquelas que tinhamaidade preconizada pararealização da mamografia foram encaminhadas posteriormente para o serviço especializado. As demais mulheres referiram satisfação com a atividade executada pela rede-escola em parceria com os serviços de saúde, o que confirma mais uma vez a efetividade da educação em saúde para detecção precoce e redução de agravos relacionados ao câncer de mama ${ }^{15}$.

Estudo realizado com 84 mulheres em sete unidades básicas de saúde (UBS), do município de Sirinhaém, Pernambuco, com cinco unidades localizadas na zona rural. A pesquisa consistia no levantamento prévio sobre os saberes das participantes, seguida de intervenção e utilização do mesmo questionário pós-intervenção. Os autores identificaram déficit de conhecimento das mulheres acerca do câncer de mama antes da execução da estratégia educativa. A conclusão do estudo foi a efetividade da educação em saúde para modificação de hábitos de saúde não condizentes com a detecção precoce do câncer de mama ${ }^{16}$. 
No que tange a utilização da SAE, enquanto método organizacional do trabalho da equipe de Enfermagem, estudo realizado com dez mulheres em instituição privada de oncologia conveniada ao Sistema Único de Saúde (SUS), em Fortaleza-CE apontou como principais diagnósticos de Enfermagem baixa autoestima, ansiedade, disposição para o conhecimento aumentada. Com isso, os profissionais melhoraram a assistência, prestando cuidado holístico, individual e humanizado, além de viabilizar a eficiência da assistência de Enfermagem ${ }^{17}$.

\section{CONSIDERAÇÕES FINAIS}

Os resultados dessa prática bem-sucedida demonstram que conhecimento baixo sobre o câncer de mama, formas de prevenção e detecção precoce pode estar correlacionado à falta de atenção e de interesse das partes, seja serviços de saúde ou usuárias. Mediante
É necessária a qualificação da equipe de enfermagem para que possam ser desenvolvidas formas que detectem precocemente o câncer de mama na atenção básica de saúde. $\mathrm{Na}$ área oncológica, verificou-se uma grande evolução da assistência de enfermagem com a melhoria do cuidado e uso de medidas que proporcionam conforto e tratamento paliativo com apoio psicológico direcionado à paciente e família. Todavia, há um longo caminho a ser percorrido para a excelência do cuidado ${ }^{14}$.

\title{
SYSTEMATIZATION OF NURSING ASSISTANCE AND BREAST CANCER IN WOMEN
}

\begin{abstract}
The breast cancer has wide incidence in the world and it is growing each day. It consists in the increase altered of cells that expand to issue and organs. This study aimed to evaluate the effect of educative intervention about breast cancer through the Systematization of Nursing Care (SNC). This is a description of the experience of a successful health practice in family health unit in community of Cabedelo city, in Paraíba State. To describe the results were followed the steps of International Classification of Nursing Practice (ICNP)®, through of seven axes. Understanding the importance of promotion the health, of the dissemination of preventive methods and early detection of breast cancer, this propose powered the professional autonomy in educational activities through of the use of SNC.
\end{abstract}

\section{KEYWORDS}

Nursing Care. Breast Neoplasms. Women.

\section{REFERÊNCIAS}

1 Rodrigues JD, Cruz MS, Paixão AN. Uma análise da prevenção do câncer de mama no Brasil. Ciênc. Saúde Colet. [Internet]. 2015. [citado em 06 mar 2018]; 20(10):3163-76. Disponível em: <http://www.scielo.br/pdf/ csc/v2on10/1413-8123-csc-20-10-3163.pdf >.
2 INCA - Instituto Nacional de Câncer José Alencar Gomes da Silva. Mama. [Internet]. 2018. [citado em 06 mar 2018]. Disponível em: <http://www2.inca.gov. $\mathrm{br} / \mathrm{wps} / \mathrm{wcm} / \mathrm{connect/tiposdecancer/site/}$ home/mama>. 
3 Gonçalves LLC, Travassos GL, Almeida AM, Guimarães AMDN, Gois CFL. Barriers in health care to breast cancer: perception of women. Rev. Esc. Enferm. USP [Internet]. 2014. [cited 2018 Mar. 06]; 48(3):394-400. Avaliable from: <http:// www.scielo.br/pdf/reeusp/v48n3/00806234-reeusp-48-03-394.pdf >.

4 OPAS/OMS - Organização Pan-Americana de Saúde/Organização Mundial de Saúde. Câncer de mama é a $2^{a}$ principal causa de morte entre mulheres nas Américas; diagnóstico precoce e tratamento podem salvar vidas. [Internet]. 2016. [citado em 06 mar 2018]. Disponível em: <http://www.paho.org/bra/index. php?option=com_content $\&$ view=article\&id=5273: cancer-de-mama-e-a-2a-principal-causa-de-morte-entre-mulheres-nas-americas;-diagnostico-precoce-e-tratamento-podem-salvar-vidas\&ltemid $=839>$.

5 INCA - Instituto Nacional de Câncer José Alencar Gomes da Silva. Síntese de resultados e comentários. [Internet]. 2018. [citado em 06 mar 2018]. Disponível em: <http://www.inca.gov.br/estimativa/2018/ sintese-de-resultados-comentarios.asp >.

6 Ministério da Saúde [Br]. Outubro Rosa alerta para o diagnóstico precoce do câncer de mama. [Internet]. 2016. [citado em 07 mar 2018]. Disponível em: <http:// www.brasil.gov.br/saude/2016/09/outubro-rosa-alerta-para-o-diagnostico-precoce-do-cancer-de-mama>.

7 Ohl ICB, Ohl RIB, Chavaglia SRR, Goldman RE. Public actions for control of breast cancer in Brazil: integrative review. Rev. Bras. Enferm. [Internet]. 2016. [cited 2018 Mar. 07]; 69(4):746-55. Avaliable from: <http:// www.scielo.br/pdf/reben/v69n4/en_00347167-reben-69-04-0793.pdf>.
8 Nascimento LKAS, Medeiros ATN, Saldanha EA, Tourinho FSV, Santos VEP, Lira ALBC. Sistematização da assistência de enfermagem a pacientes oncológicos: uma revisão integrativa da literatura. Rev. Gaúcha Enferm. [Internet]. 2012. [citado em 08 mar 2018]; 33(1):177-85. Disponível em: <http://www.scielo.br/pdf/ rgenf/v33n1/a23v33n1.pdf>.

9 Nascimento KTS, Fonsêca LCT, Andrade SSC, Leite KNS, Costa TF, Oliveira SHS. Sentimentos e fontes de apoio emocional de mulheres em pré- operatório de mastectomia em um hospital-escola. Rev. Enferm. UERJ [Internet]. 2015. [citado em 08 mar 2018]; 23(1):108-14. Disponível em: <http://www.facenf.uerj.br/v23n1/ v23n1a18.pdf $>$.

10 Gutiérrez MGR, Morais SCRV. Systematization of nursing care and the formation of professional identity. Rev Bras Enferm [Internet]. 2017 [cited 2018 Mar. 13]; 70(2):436-41. Avaliable from: <http:// www.scielo.br/pdf/reben/v70n2/00347167-reben-70-02-0436.pdf >.

11 ICN. International Council of Nurses (ICN). About ICNP®: definitions \& elements [Internet]. Geneva; 2010.

12 Oshiro ML, Silva RG, Costa KC, Travaim IEB, Silva GB, Thuler, LCS. Câncer de mama avançado como evento sentinela para avaliação do programa de detecção precoce do câncer de mama no Centro-Oeste do Brasil. Rev Bras Cancerol. [Internet]. 2014. [citado em 15 mar 2018]; 60(1):15-23. Disponível em: <http://www.inca.gov.br/rbc/n_60/v01/ pdf/04-artigo-cancer-de-mama-avancado-como-evento-sentinela-para-avaliacao-do-programa-de-deteccao-precoce-do-cancer-de-mama-no-centro-oeste-do-brasil.pdf>. 
13 Nascimento KTS, Fonsêca LCT, Andrade SSC, Leite KNS, Zaccara AAL, Costa SFG. Multi-professional team care: discourse of women in preoperative mastectomy. Esc Anna Nery [Internet]. 2014. [cited 2018 Mar. 15]; 18(3):43540. Avaliable from: <http://www.scielo.br/pdf/ean/v18n3/en_1414-8145ean-18-03-0435.pdf>.

14 Moraes DC, Almeida AM, Figueiredo EN, Loyola EAC, Panobianco MS. Rastreamento oportunístico do câncer de mama desenvolvido por enfermeiros da Atenção Primária à Saúde. Rev Esc Enferm USP [Internet]. 2016 [citado em 13 mar 2018]; 50(1):14-21. Disponível em: < http://www.scielo.br/pdf/reeusp/v50n1/ pt_0080-6234-reeusp-50-01-0014.pdf>.

15 Silva RM, Melo DC, Barbosa SEB, Silva JMO, Batista FMA. Educação em Saúde para prevenção do câncer de mama no município de Piripiri-PI: atuação do petsaúde. R. Epidemiol. Control. Infec. [Internet]. 2015. [citado em 14 mar 2018]; 5(4):203-5. Disponível em: <https://online.unisc.br/seer/index.php/epidemiologia/article/download/5458/4632>.

16 Bushatsky M, Cabral LR, Cabral JR, Barros MBSC, Gomes BMR, Filho ASSF. Health education: a strategy for action against breast cancer. Ciênc. Cuid. Saúde [Internet]. 2015. [cited 2018 May. 14]; 14(1):870-8. Avaliable from: <http://periodicos.uem.br/ojs/index.php/CiencCuidSaude/article/view/23259/pdf_288>.

17 Oliveira SKP, Viana MTMP, Bilhar SPO, Lima FET. Sistematização da assistência de enfermagem às mulheres mastectomizadas. Cogitare Enferm. [Internet]. 2010. [citado em 15 mar 2018]; 15(2):319-26. Disponível em: <http://revistas.ufpr.br/cogitare/article/view/17869>. 